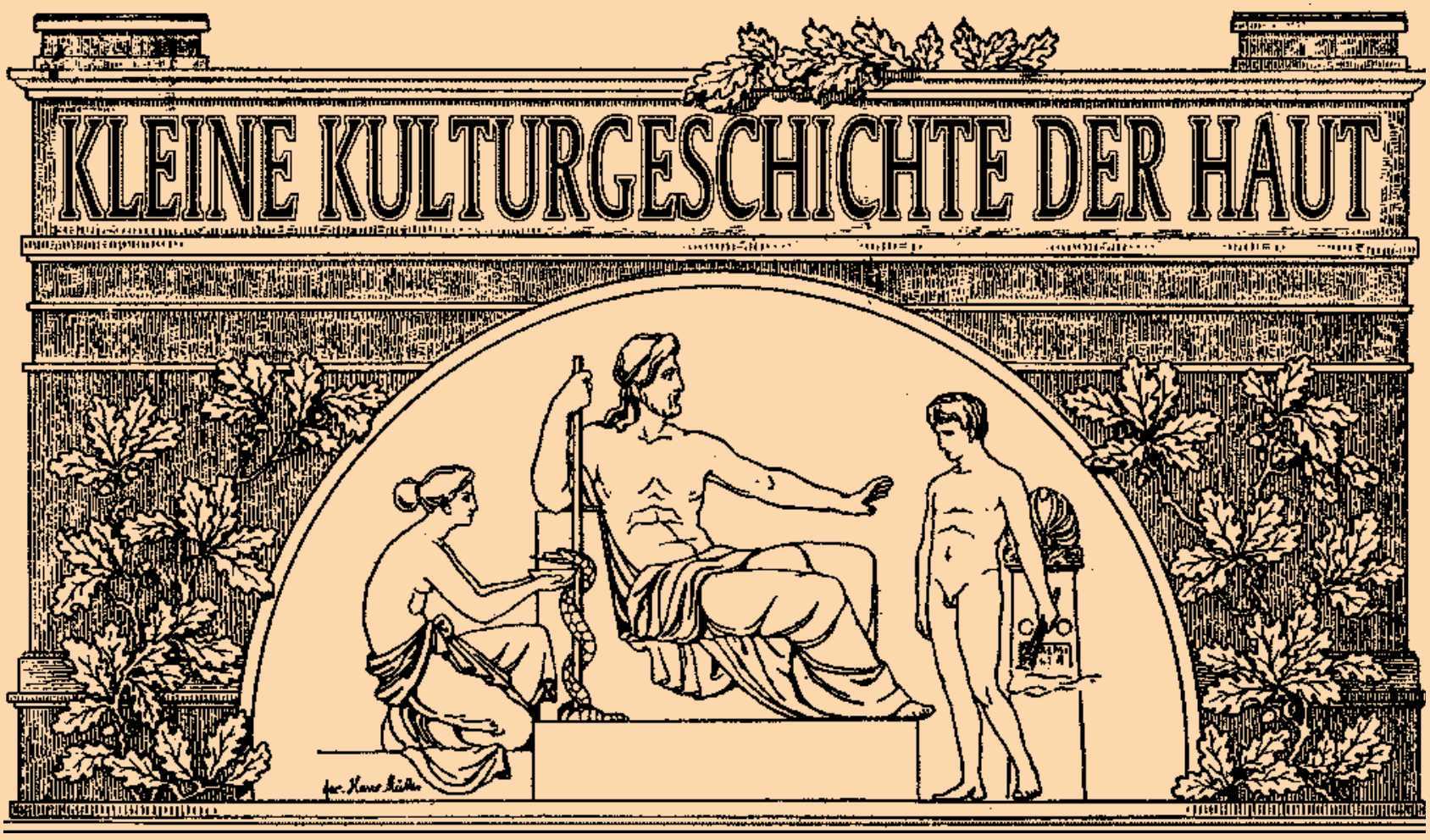

Die Zeitschrift „Aktuelle Dermatologie“ hat seit Januar 2004 bis zu diesem Heft regelmäßig einen kurzen, informativen und prägnanten Beitrag $z u$ dieser Rubrik veröffentlicht. In den drei Jahren sind mehr als 30 Beiträge zusammengekommen. Diese sind von den Lesern sehr gut angenommen worden, wie Zuschriften und viele Gespräche bezeugen. Ideen wurden mir reichlich angetragen und Kollegen haben sich als Autoren zugesellt. Es hat Freude bereitet, diese Rubrik zu konzipieren und zu verwirklichen. Dabei möchte ich allen Mitautoren herzlich danken für ihren Enthusiasmus, ihre Originalität und ihre Schaffenskraft. Der Plan besteht, die Reihe zusammenzufassen und als Buch herauszugeben. Nun aber kommt diese Rubrik hier zum Ende, resp. sie wird gefolgt von einer neuartigen Sequenz. Lassen Sie sich von der neuen, die Schrift führenden Herausgeberin Christiane Bayerl überraschen.

Ihr Ernst G. Jung

\title{
Die weiße Dame von Abri Maak
}

\author{
The White Lady from Abri Maak
}

Bibliografie

DOI $10.1055 / \mathrm{s}-2006-944939$

Akt Dermatol 2006; 32;

533-535 @ Georg Thieme

Verlag KG Stuttgart · New York

ISSN 0340-2541

Korrespondenzadresse Dr. Manfred Reitz

Schillerstr. 7

99423 Weimar

mreitz@fli-leibniz.de
Eine dunkle Haut ist kein rassenspezifisches Merkmal, sondern eine Anpassung an die UV-Bestrahlungsintensität des jeweiligen Lebensraumes. Einerseits ist der menschliche Organismus auf eine gewisse UV-Bestrahlung angewiesen, andererseits darf diese UV-Bestrahlung jedoch nicht zu hoch ausfallen, um keine Schädigungen auszulösen. UV-Strahlen regen zum Beispiel die Synthese von Vitamin D an und sind somit lebenswichtig. Gleichzeitig zerstören UV-Strahlen aber auch in den feinen Blutgefäßen der Lederhaut die für den Zellstoffwechsel wichtige Folsäure, so dass sich ein Folsäuremangel entwickeln kann. An Ratten und Mäusen wurde gezeigt, dass Folsäuremangel die Fruchtbarkeit beeinträchtigt und sich somit die Überlebensrate in einer Population sofort gegen Individuen mit Folsäuremangel richtet. Da UV-Strahlen außerdem die DNA der Hautzellen schädigen, fördert eine $\mathrm{zu}$ hohe UV-Strahlenbelastung zusätzlich noch die Hautkrebsentwicklung. Individuen mit einer für ihre Umwelt falsch angepassten Hautfarbe sterben deshalb aufgrund der UV-Bestrahlungsfolgen langfristig aus [1].

\section{Anpassungen der Haut an Bestrahlungsbelastungen}

Die menschliche Haut reagiert durch Pigmenteinlagerungen auf die aktuell vorherrschende UV-Strahlenbelastung und stellt sich auf ein 


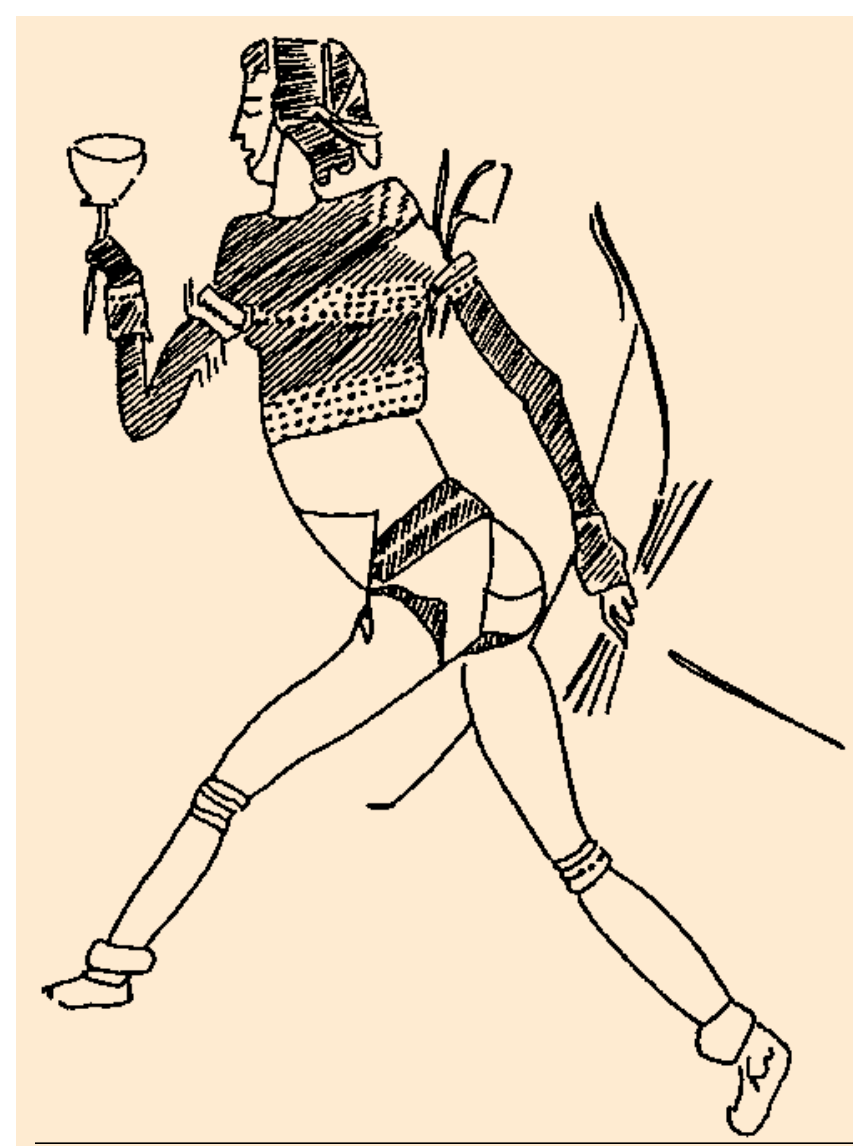

Abb. 1 Die „weiße Dame“ von Abri Maak, Felsmalerei (Namibia), um 5000 v. Chr. Die dargestellte Person sieht nicht wie eine Negride aus, sie trägt Schuhe, enge Kleidung und ein Haarnetz.

Gleichgewicht zwischen den jeweiligen Vor- und Nachteilen der allgemeinen Bestrahlungsdosis ein. Bei Tieren übernimmt das Fell eine solche Schutzfunktion. Schimpansen, die genetischen Vettern des Menschen, besitzen unter ihrem Fell eine helle Haut und sind nur in Körperbereichen ohne Fell dunkel pigmentiert. Vermutlich haben deshalb die Vorläuferformen des Menschen erst nach dem Verlust ihres Fells eine variable Hautpigmentierung entwickelt und konnten dadurch an ihren afrikanischen Entwicklungsorten überleben. Als sich der Mensch später über die Erde verteilte, musste er immer wieder seine Hautfarbe an die vorherrschende UV-Strahlenbelastung seines Lebensraumes anpassen. Sind Weltgegenden von der Sonne durchflutet, leben dort auch in der Gegenwart überwiegend dunkelhäutige Menschen, während Weltgegenden, die von der Sonne wenig verwöhnt sind, meist von hellhäutigen Menschen besiedelt werden.

Frühe Wanderbewegungen von Menschen können häufig über die Hautfarbe der Besiedlungspioniere rekonstruiert werden. Eigentlich besitzen Eskimos (Inuit) für den kalten Norden eine viel zu dunkle Hautfarbe. Der Grund ist einfach: Da sie erst vor weit weniger als 5000 Jahren aus Asien in den sonnenarmen Norden eingewandert sind, sind sie in ihrer Hautfarbe noch nicht optimal an ihre Umwelt angepasst. Außerdem ernähren sie sich praktisch nur von tierischen Materialien und verfügen deshalb über eine besonders Vitamin-D-reiche Kost, ihre eigene Vitamin-D-Produktion kann ohne Schädigungen minimal gehalten werden. Sie müssen deshalb nicht rasch eine helle Hautfarbe annehmen. Im Süden des Sudan ist die Hautfarbe der einheimi- schen Bevölkerung besonders dunkel. Auf der in der Sonnenintensität vergleichbaren südlichen Arabischen Halbinsel ist die Hautfarbe der einheimischen Bevölkerung dagegen weitaus heller. Diese Menschen sind arabischer Abstammung und erst vor etwa 2000 Jahren zugewandert. Anpassungsstrategien könnten bei ihnen noch im Gange sein. Zusätzlich bremsen bei ihnen kulturelle Verhaltensweisen die Anpassung der Hautfarbe an eine hohe UV-Strahlenbelastung. Araber schützen sich durch eine dichte Kleidung vor den UV-Strahlen ihrer Umwelt und lebten zumindest früher ständig in schützenden Zelten. Heute leiden in Schottland viele der erst jüngst eingewanderten, dunkelhäutigen Inder auffallend häufig an Rachitis und anderen fast vergessenen Vitamin-D-Mangelerkrankungen. Ihr Organismus kann im Gegensatz zu den hellhäutigen Einheimischen aufgrund der dunklen Hautfarbe nicht genügend Vitamin D selbst herstellen, und sie gleichen möglicherweise durch die Übernahme der Ernährungsgewohnheiten der Einheimischen ihren Vitaminmangel nicht vollständig durch geeignete Nahrungsmittel aus. Hellhäutige Europäer erkranken gegenwärtig in Nordaustralien überdurchschnittlich oft an Hautkrebs, während die gleichen Krebsarten bei den in der Hautfarbe angepassten Aborigines relativ selten vorkommen [1].

\section{Frühe hellhåutige Menschen im südlichen Afrika $\nabla$}

Prähistorische Bilddarstellungen können in manchen Weltgegenden echte Überraschungen hervorrufen und zeigen, dass beispielsweise vor mindestens 7000 Jahren hellhäutige Menschen in das südliche Afrika eingewandert sind und sich dort möglicherweise später in ihrer Hautfarbe genetisch an ihren neuen Lebensraum angepasst haben. Der deutsche Landvermesser Reinhard Maak entdeckte 1917 etwa 300 km nordwestlich von Windhuk im damaligen Deutsch-Südwestafrika (heute Namibia) an einem Felsüberhang höchst merkwürdige Malereien. Der Felsüberhang, auch Abri genannt, erhielt später seinen Namen. Dargestellt waren dort hellhäutige und oft rothaarige Menschen bei einem feierlichen Umzug. Insbesondere eine „weiße Dame“ fiel auf, die sich in ihrem Aussehen völlig von typischen Darstellungen afrikanischer Ureinwohner unterschied [2]. Die auf dem Felsen abgebildete feingliedrige Frau trug Schuhe, ein Haarnetz sowie eng anliegende Kleidungsstücke ( $\bullet$ Abb. 1). In einer Hand hielt sie einen Bogen mitsamt Pfeilen und in der anderen Hand eine Lotosblüte. Stilistisch und in der Eleganz der Bewegung verwies die Malerei in den fernen Mittelmeerraum und verriet Anklänge an frühe altägyptische Darstellungen. Spätere Datierungen zeigten, dass die Bilder etwa um 5000 v. Chr. gemalt worden waren.

Über die Herkunft und das weitere Schicksal der hellhäutigen Menschen im südlichen Afrika wird bis heute gerätselt. Noch am Ende der Steinzeit war die gegenwärtige Wüste Sahara grün und glich einer fruchtbaren Savannen- und Steppenlandschaft mit großen Seen, zahlreichen Flüssen sowie einer reichen Tierwelt. Vor ungefähr 6500 Jahren lag zum Beispiel der Wasserspiegel des Tschad-Sees rund $40 \mathrm{~m}$ höher als in der Gegenwart und ähnelte eher einem Binnenmeer als einem See. Beiderseits des Mittelmeeres lebten während dieser Zeit sowohl auf europäischer als auch auf nordafrikanischer Seite Cro-Magnon-Menschen, die sich nach ihren Skelettfunden von modernen Europiden unterschieden, aber wie diese hellhäutig waren. Als später die Sahara immer trockener und lebensfeindlicher wurde, mussten ihre Bewohner auswandern und neue Lebensräume erschlie- 


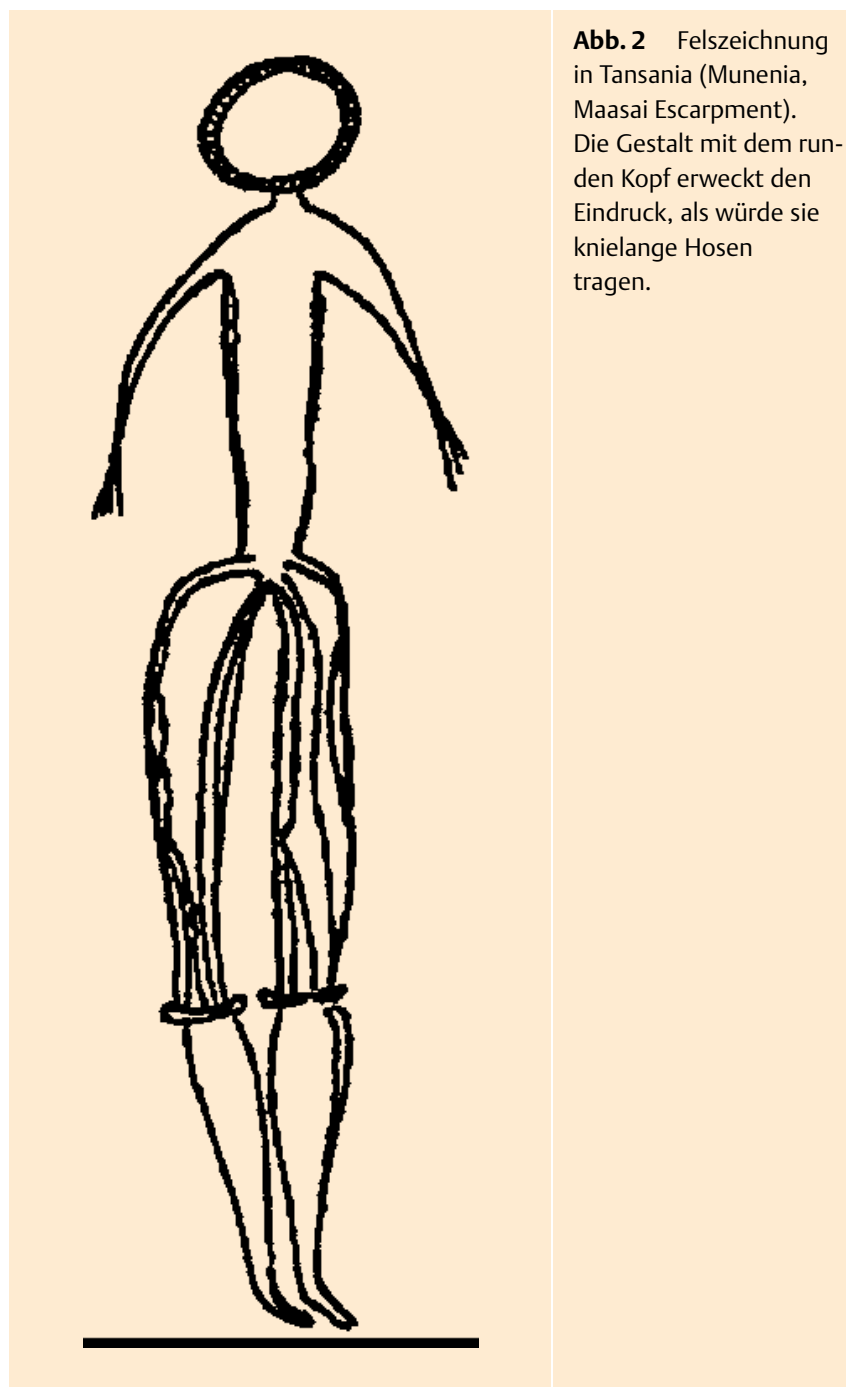

ßen. Einige machten die Sümpfe des Niltals fruchtbar und gründeten eine der ersten Hochkulturen. Andere wichen tief in den afrikanischen Süden aus und schufen dort heute noch weitgehend unerforschte Kulturen. Das weitere Schicksal dieser hellhäutigen Siedler ist unbekannt. Möglicherweise haben sie genetisch ihre Hautfarbe an die UV-Strahlenbelastung ihrer neuen Heimat angepasst und wurden mit der Zeit dunkelhäutig. Während der Steinzeit gab es nach der Analyse von Skelettfunden in Afrika noch keine Menschen der negriden Großrasse. Diese frühen Afrikaner waren zwar als Reaktion auf die UV-Strahlenbelastung dunkelhäutig und stammten direkt von den ersten Vertretern des modernen Homo sapiens ab, aber sie waren keine Negride. Im südlichen Afrika lebten während der mittleren Steinzeit mindestens drei unterschiedliche Menschenrassen, die außer der Hautfarbe alle nicht typisch negrid aussahen. Aus Varianten dieser Rassen entwickelten sich später die heutigen Khoisaniden, die Völker der Buschmänner und Hottentotten. Insbesondere in Ostafrika gab es noch am Ende der Steinzeit hoch gewachsene Menschen, deren Schädelform stark dem europäischen Cro-Magnon-Typ entsprach. Die heutigen Negriden sind im Gegensatz zu ihnen jüngere Anpassungen an ein heißes und lichtintensives Klima. Sie haben sich vermutlich erst nach der Steinzeit zuerst in Westafrika entwickelt. Frühe Skelettfunde von Menschen mit typischen negriden Merkmalen tauchten erstmals nach dem Ende der Steinzeit auf. Die anschließende erfolgreiche Ausbreitung der Negriden in Afrika setzte erst relativ spät mit der Metallverarbeitung und dem Ausbau der Landwirtschaft ein $[3,4]$.

Frühe Jäger- und Sammlerkulturen im südlichen Afrika unterschieden sich unter anderem auch in der Kleidung der Menschen von den späteren Kulturen der Negriden. Uralte und schwierig zu datierende Felsmalereien in Tansania zeigen beispielsweise oft menschliche Gestalten, die den Eindruck erwecken, als würden sie Hosen tragen, die ihnen bis an die Knie reichen ( Abb. 2). In späteren Epochen und nach der Ausbreitung der Negriden kamen solche Kleidungsstücke im südlichen Afrika nicht mehr vor [5].

\section{Weitreichende frühe Kulturkontakte? \\ $\nabla$}

Felsmalereien in Höhlen und an Felsüberhängen reichen in einem gewaltigen Bogen vom südlichen Afrika durch die ehemals grüne Sahara bis nach Westeuropa. Im südlichen Afrika wird das Alter mancher Malereien auf rund 26000 Jahre geschätzt. Einige Forscher vermuten sogar, dass sich trotz der großen Entfernungen diese Malereien und Reliefdarstellungen von Europa bis nach Afrika gegenseitig beeinflusst haben, und die Menschen untereinander in lockeren kulturellen Kontakten gestanden hatten. Für manche Stilrichtungen gibt es vor Ort keine nachweisbaren Entwicklungswege und sie erscheinen wie aus dem Nichts plötzlich auf einem hohen künstlerischen Niveau. Die Tierdarstellungen in der Höhle von Lascaux in Frankreich werden wegen ihrer Qualitäten als die „Sixtinische Kapelle der Eiszeit“ bezeichnet. Picasso soll nach einer Besichtigung tief beeindruckt berichtet haben: „Nichts haben wir erfunden, nichts!“ Der kulturelle Standard der Steinzeit war vermutlich weltweit weitaus höher entwickelt als allgemein angenommen. Da es sich um schriftlose Kulturen handelt, fehlen allerdings heute die Dokumente und es sind bei einem sehr lückenhaften Beweismaterial nur indirekte Rückschlüsse möglich [2,4-6].

\section{Literatur}

1 Jablonski NG, Chaplin G. Die Evolution der Hautfarbe. Spektrum der Wissenschaft 2003; 38-44

2 Wendt $H$. Ich suchte Adam. Die Entdeckung des Menschen. Hamburg: Rowohlt, 1966

3 Knußmann R. Vergleichende Biologie des Menschen. Stuttgart, Jena: Gustav Fischer, 1996

4 Connah G. Unbekanntes Afrika. Archäologische Entdeckungen auf dem Schwarzen Kontinent. Stuttgart: Theiss, 2006

5 Anati E. Höhlenmalerei. Die Bilderwelt der prähistorischen Felskunst. Zürich, Düsseldorf: Benzinger, 1997

6 Scarre C (ed). The Human Past. London: Thames \& Hudson, 2005 